\title{
A New View on Bacterially-Produced Uraninite Nanoparticles Ultrastructure Revealed with Aberration Corrected STEM.
}

\author{
Alice C. Dohnalkova ${ }^{1}$, Arda Genc ${ }^{2}$, Kenneth M. Kemner ${ }^{3}$ and William D. Burgos ${ }^{4}$ \\ 1. Environmental Molecular Sciences Laboratory, Pacific Northwest National Laboratory, Richland, WA \\ 2. FEI Company, 5350 NE Dawson Creek Drive, Hillsboro, OR 97124, USA \\ 3. Biosciences Division, Argonne National Laboratory, Argonne, IL \\ 4. Department of Civil and Environmental Engineering, The Pennsylvania State University, University \\ Park, PA
}

Uranium in contaminated aquifers poses a serious environmental threat for soils and groundwater at present and in the future. One of the strategies for $U$ immobilization in soils is microbiologicallymediated reduction of soluble $\mathrm{U}(\mathrm{VI})$ to a relatively insoluble $\mathrm{U}(\mathrm{IV})$, uraninite $\mathrm{UO}_{2}(\mathrm{~s})$. The reduction of uranium(VI) by Shewanella oneidensis MR-1 was studied to examine the effects of bioreduction kinetics on the physical properties and susceptibility to re-oxidation of the biogenic $\mathrm{UO}_{2}$ [1]. Biogenic U(IV) precipitates were characterized by high resolution electron microscopy and synchrotron X-ray absorption spectroscopy methods.

S. oneidensis MR-1 cells were exposed to uranyl acetate as the electron acceptor and sodium lactate as the electron donor under resting cell conditions [1]. In addition to bioreduction kinetic studies, ultrathin sections of plastic-embedded cultures were studied by high resolution transmission electron microscope (HR TEM), and correlated synchrotron-based X-ray studies were conducted.

According to the HR TEM imaging and analysis, the average size of the biogenic $\mathrm{UO}_{2}$ nanoparticles was $\sim 3.0 \mathrm{~nm}$. Extended X-ray absorption fine-structure spectroscopy (EXAFS) was also used to estimate uraninite particle size based the U-U coordination number in $\mathrm{UO}_{2}$. It suggested a particle size of a comparable size with the TEM measurements. However, in different experimental conditions, the EXAFS was able to show a slight trend of smaller particle sizes with faster reduction rates, with particles estimated to be of a smaller diameter, approximately $1.2 \pm 0.8$ and $1.5 \pm 0.8 \mathrm{~nm}$, respectively, which is significantly less than the TEM result of $\sim 2.9 \mathrm{~nm}$.

During the opportunity to re-examine the thin sections using the aberration corrected Scanning TEM (STEM, FEI Titan ${ }^{\mathrm{TM}}$ ), surprisingly, a variety of smaller size particles appeared to be present, ranging from very small organized associations consisting of only a few tens of atoms, to the particles sizes documented previously. This unprecedented resolution opened a whole new view onto particulate ultrastructure, but it also showed in remarkably improved detail the occurrence of condensed particles described previously based on the HRTEM.

The aberration corrected high angle annular dark field (HAADF) STEM imaging enabled the unparalleled atomic-level imaging of newly formed bacterially-induced biominerals. This newly acquired information will provide a base for hypotheses about the reductive formation and/ or reoxidation of these nanoparticles and particle aggregation state, including work on understanding the nanoparticulate uraninite and monomeric uranium formation, with important implications in the research studying the transport and fate of heavy metals and radionuclides in the environment [2, 3, 4]. 


\section{References:}

[1] WD Burgos et al, Geochimica et Cosmochimica Acta 72 (2008), 4901-4915.

[2] MI Boyanov et al, Environ. Sci. Technol. 45 8336-8344, 2011.

[3] KE Fletcher et al, Environ. Sci. Technol. 44 4705-4709,2010.

[4] V. Sivaswamy et al, Biotechnol. Bioeng. 108 264-276, 2011.

[5] This research was performed at the Environmental Molecular Sciences Laboratory, a national scientific user facility sponsored by the U.S. DOE, located at PNNL.

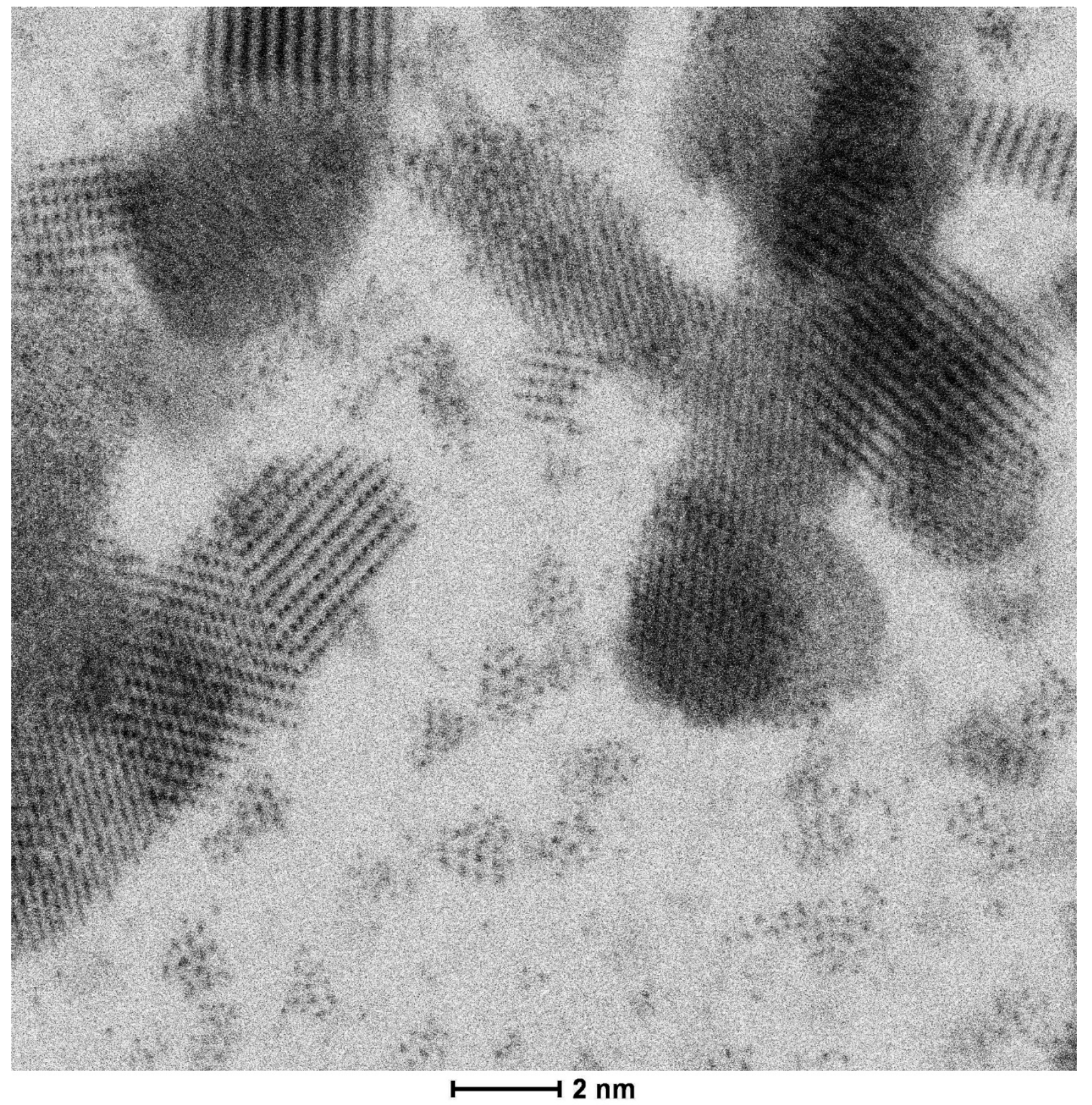

Figure 1. STEM image of biogenic $\mathrm{UO}_{2}$ particles formed as a result of reduction of U(VI) solution by bacterium Shewanella oneidensis MR-1. The unprecedented resolution enabled to visualize previously unresolved sub-nanometer particles that play important role in predicting the system function. 